was caused by renewed attempts at the taxis. Cold applications had been fully tried previously. At length the operation was proposed to the patient, who readily consented. Layer after layer were accordingly cut through, till what seemed to be intestine was laid bare. The stricture part of the ring was divided, but still the intestine could not be reduced. The wound was dressed, a few drops of laudanum given, and the patient left to rest.

19th.- - Iaving some misgivings that all was not right, I hastened to see my patient in the morning. He was no worse, but vomiting and hiccup still troubled him, and the bewels were not open. I removed the dressings from the wound, and on examining minutely, found what seemed to be intestine to be composed of two layers. I therefore cut a small portion carefully out of the external one, when about two ounces of serum issued out. The hernial sac was now divided, in different directions, with the aid of the director and probe-pointed bistoury; the stricture, which seemed to be at the internal ring, was sufficiently divided, and about five inches of intestine were returned. From this time all the bad symptoms subsided, the wound healed favourably, and in two weeks the patient was able to sit up, and walk about his room.

Having never had an opportunity of seeing the operation performed by another, it will not be thought strange if an error was committed: it was an error on the safe side, and fortunately led to no bad results. I have to thank Dr. M. S. Buchanan, of Glasgow, for the instructions he gave when I was his pupil-particularly as to rubbing the sac between the fingers, to separate it from the intestine. My former patient now wears a truss, is in excellent health, and more robust than ever.

Retention of Urine.-G. B-, labourer, Goodwill, aged sixty; short stature; thin habit; has complained several years of frequent calls to make urine, with difficulty in passing it. Stricture was suspected, but he never had any treatment for it. On the 13 th of November, I was sent for to see him, at his provision ground, six miles' distance, being informed that he had retention since the previous evening. Providing myself with catheters of different sizes, 1 went to see him. He was suffering extreme pain, and the retention was complete. The distended bladder could be felt above the pubes. A warm bath and repeated opiates were given without effect. With some difficulty, a very small silver catheter was introduced, and the urine withdrawn. A dose of castor oil was ordered, and the catheter left in the bladder.

14 th.-On returning this morning, I found that the catheter had slipped out, not having been properly secured. The calls to pass urine were as urgent as ever. All attempts to introduce the instrument now failed, and a discharge of blood from the urethra showed that too much force had already been used. I now got him conveyed home in a gig. The road being rough, much suffering was occasioned. Another attempt to pass the catheter having failed, as well as warm baths, I punctured the bladder an inch and a half above the pubes with a hydrocele trocar, having no other convenient instrument. I straightened a large male silver catheter, and introduced it through the canula, which was now withdrawn, for fear it might be too short. Forty drops of solution of merphia were given.

15th.-Had a good night; urine passes freely through the tube. Ordered, castor oil, an ounce.

16th.-Finds the catheter troublesome, as, from its length, it falls to one side when he is on his back; a considerable enlargement of the prostate discovered.

18th.- The catheter has fallen out, the urine again accumulating. A female catheter was now substituted as a tube, an opening having been made for it with the lancet; no urine passed by the urethra.

20th.-The female catheter, with a stopper, seems to answer very well, only that he thinks the end of it hurts the posterior side of the bladder; it is well secured with tapes, fastened to a handkerchief, which is fastened round the body. No bongie or catheter can pass through all the extent of the urethra: they go through two strictures at the bulb, but cannot be got throngh the prostatic portion of the urethra though instruments of different sizes and curvatures are used To take castor oil occasionally.

Dec. 3rd.-Walks about a little; still no urine per urethram.

13th.-The natural passage is now restored-probably from the subsidence of the inflammation of the internal parts. The tube was withdrawn, and the artificial opening, being covered with a strip of plaster, gradually closed. In two weeks after, the patient was rapidly regaining his strength, and expressed much gratitude on account of his recovery.
Retention of Placenta.-A. D-, Orange Valley, aged twenty-two, mulatto; October, 1816 ; first labour. In this case the child was delivered easily, but the placenta retained. I saw the patient eight hours after, and endeavoured to extract the placenta by gentle traction at the cord, at the same time endeavouring to get the fingers within the os uteri. This was found impracticable. Several doses of ergot of rye were given without effect. Next day, attempts were again made to dilate the constricted portion of the uterus, without avail; only two fingers could be introduced so as to touch the internal surface of the placenta. Irritative fever came on, and the patient sank three days after delivery.

No post-mortem examination was allowed, the relatives arguing that she had been sufficiently examined already. This is the only case of retained placenta I have met with in Jamaica. Parturition, generally speaking, is attended with very few accidents in this favoured clime, and a medical man is seldom required.

Amputations.-Within three years and a half, I have had six cases: five were for sores of the legs. Mary R-, at Lima, aged forty, had had sores for twenty years; the fibula was divided into two portions, and the tibia half eaten through with caries. A speedy recovery followed.

$\mathrm{C}-$ Paisley estate, aged thirty five, had a sore on the shin, which had annoyed him seven years, and could not be cured. On examining the amputated limb, after the operation, the tibia was found carious in the same manner. A quick recovery followed.

F-_- from Maldon, mulatto, carpenter, aged forty; had disease of the ankle.joint for many years. The patient seemed in a fair way of recovery the first ten days, when secondary hæmorrhage came on, which could not be arrested. He sank two days after.

W. J-, Orange Valley, aged eighteen, had oedema of foot and ankle, in consequence of a sprain two years previously. After the use of a stimulating embrocation, gangrene commenced, and amputation became indispensable. He has had sores on the other foot since, but is well in other respects.

Catherine Q - Deeside, aged eight years, had a sore on one foot, which commenced from the toes, afterwards becoming gangrenous, and extending to the ankle. The operation was deferred by the relatives till the patient was too low. Death followed next day.

All these operations were performed with the doubleflap, five inches from the knee.

L_ at Adelphi estate, aged twelve, got his hand and wrist crushed in a sugar-mill. The double-flap operation was performed below the elbow, and a cure speedily effected.

Croup.-Only two cases have occurred in my practice:-

$\mathbf{R - C}$, a negro child from Amity Hall, ten miles distance. The difficulty of breathing was great, and the hoarse, brazen congh could not be mistaken. The treatment consisted of antimonial emetics, calomel purges, blistering, and the warm bath. Recovery soon followed.

Charles $R$-, a mulatto child, had the same symptoms, and being actively treated, soon got well.

I have detailed above a few of the more prominent cases which have occurred within less than four years. If this should meet the eyes of any young student or surgeon intending to go abroad, let him devote every opportunity, while he is in Europe, to perfect himself in anatomy, midwifery, practical surgery, and other branches of medical science, as for all he must in most cases depend on himself and his previous study, when settled in a distant region.

\section{ON THE EMPLOYMENT OF TARTAR-EMETIC IN CHOLERA.}

By C. RADCLYFFE HALL, M.D., M.R.C.S., \&c., Holmes Chapel.

Amongst the fourteen modes of treatment of Asiatic cholera enumerated by Mr. Gower, in The LanceT for August 5th, I find no reference to what $I$ believe to be a mode superior to all the rest-the treatment by large doses of tartarized antimony. From the deprecatory allusion to emetics, (see $\$ 9$, p. 155, it is probable that, in theory, this plan would hardly meet with MIr. Gower's sanction; and as I find, on referring to two works very likely to be consulted-viz., the "Library of Medicine," and the "Dictionary of Medicine," that it is unnoticed both by Dr. Copland and by Dr. George Budd, I am induced to address you on the subject.

Evidently an off'shoot of Rasori's contra-stimulant doctrine, the emetic treatment of Algide cholera by tartarized anti- 
mony, originated in Italy, was noticed in a current number of Dr. Johnson's Medico-Chirurgical Review. It there came under the observation of my friend, Mr. Stott, of Manchester, by whom, and by others, at his suggestion, it was very extensively tried at the cholera hospitals, and in private practice, during the invasion of 1832 .

My own experience of its efficacy is founded entirely on observation of the practice of Mr. Stott, with whom at that time I had the pleasure of residing. I have notes of merely the first eight cases. Of these, only one proved fatal. In this instance, diarrhœa had existed for four days previous to the supervention of vomiting and collapse, and had been treated with calomel and opium; the patient obstinately refused to drink freely, the antimony caused vomiting but twice, and collapse increased unintermittingly to the end.

The ordinary mode of proceeding was as follows:-Five grains of tartarized antimony were dissolved in half a pint of camphor mixture; of this an ounce was given every two hours. The patient was urged to drink freely of toast-water. Immediately, or in a short time after the first dose, vomiting occurred, and was encouraged by the toast-water. After a time, the patient usually objected to the copious drinking of the water, and required much urging to persevere. Perseverance, however, was strongly enjoined. It was noticed in nearly all the fatal cases, that the objection to continued drinking had not been overcome. This plan was unceasingly persevered with, presenting a scene of incessant drinking and throwing up, until the stomach became tolerant of both the antimony and the fluid. The mixture was then continued, with less drinking of toast-water, so long as the symptoms required it. Simultaneously with, or shortly after the cessation of, vomiting, the symptoms usually improved. The good signs were these-warmth of tongue; gentle warm perspiration, and secretion of urine; increased volume of pulse, subsidence of cramps and of diarrhœa, and less of the extreme dejection of mind: sometimes, a little bile in the matter vomited, or a bilious stool. The antimony was now discontinued, the patient allowed to remain quiet, warmth applied to the feet, and a little arrow-root gruel given, flavoured or not with brandy, according to circumstances. The patient generally fell into a quiet sleep, and awoke feeble, but feeling comparatively well and in good spirits. The action of the bowels was next attended to by giving castor oil, and very little other than hygieftc treatment was needed subsequently. When the cramps attacked the abdomen, sinapisms were applied until the effect of the antimony had had time to remove them.

The advantages of this treatment were found to be- 1 , its superior efficacy as a means of cure; 2 , its speedier operation in curing; 3 , the absence of the fever of reaction, with its accompanying risk of pneumonia and other serious lesions-a result not ordinarily attending any of the other methods of treatment.

The rationale of this treatment-homœopathic in theory, heroic in practice-is easily deduced from the known pathology of cholera. All we know of the facts of cholera is summed up in few words. After exposure to the influence, the subject sickens, there is extreme prostration of mind and body, shock of all parts of the system, a check to all natural secretion, inability on the part of the vascular organs to circulate the blood properly, want of blood on the surface, superabundance of blood in the viscera, a gorged state of the internal veins, effusion of the serous part of the blood through the gastrointestinal mucous membrane, and, consequently, serous vomiting and purging, deficient animal heat and cramps. What, then, are the indications?

1. To restore the circulation by dislodging the gorged internal vessels of their contents, by sending blood to the surface, and so to gain time.

2. To follow Nature's indication of ejecting the materies morbi, without allowing death to ensue in the meantime; or ortherwise to follow, to a safe extent, Nature's own plan of action, until the morbid agency ceases to exercise its deleterious influence.

3. To restore fluid to the drained and inspissated blood.

4. To restore secretion.

Theoretically, we might have doubted the safety of exhibiting a prostrating remedy like tartar emetic in a disease attended with prostration, like cholera. On such a principle we should not bleed in peritonitis. But the prostration of cholera is kept up by the almost stagnant circulation in the capillaries, and the engorgement of the veins, conditions which antimony, when absorbed, has a tendency to obviate, and which, as an emetic, both mecharically and through the ganglionic nerves, it tends to overcome. The distinction between spontaneous vomiting and vomiting artificially induced must be borne in mind. The former exhausts far more rapidly and seriously than the latter. Tartar emetic never kills by vomiting. However, the discussion of the principle of administration is of very secondary importance to the practice, and it is on practical, and not on theoretical grounds, that I suggest a trial of the plan, should the expected opportunity occur.

It is well to bear in mind the points we do not know in the pathology of Algide cholera.

1. The nature of the morbid poison, whether electric, animalcular, malarial, or animal.

2. The laws of its transmission, whether as an epidemic only, or as an epidemic and infectious disease also.

3. The laws of its operation, whether imbibed into the blood through the respiratory and rascular mucous tract generally, or through the skin, or whether it act in some indescribable manner on the nervous system without necessarily boing received into the blood at all. Assuming its reception into the circulation in the first instance--whether it merely mixes with the blood and suspends secretion and muscular power by its own action on secreting cells and muscular fibre, and nervous power by a similar action on nerve-cells; or affects secreting and muscular tissues only through the medium and intervention of the nervous system to which its direct influence, whilst circulating in the capillaries, is restricted; whether it changes the quality of the blood like the typhoid poison prior to acting on the solids at all, or whether it performs all these actions at one and the same time.

Whether the blood or the nervous centres take the lead in causing the phenomena,--whether the vitiated blood stops secretion, disorders the nerves, and irritatos the muscles, or the checked secretion causes the accumulation of vitiating matter in the blood,- - whether the serous discharge be a morbid secretion set up for the ejection of the morbid poison, or a mere transudation of the thinner parts of the blood from the distended portal venous system,-in practice we scarcely require the information to guide us. We have vitiated blood, internal congestion, torpid nervous energy, feeble heart checked secretions, and exhausting discharge, to contend with, whatever their mode or sequence of causation.

Many practitioners are sarcastic at the expense of the prescriber of " hot and cold in the same breath," and criticise unmercifully such a combination as that of ipecacuanha and dilute sulphuric acid. It may be worth considering notwithstanding, whether in cases of cholera attended with extreme collapse and want of pulse, the antimonial treatment might not advantageously be conjoined with the exhibition of ammonia, ether, brandy, or capsicum, the hot-air bath, or other internal or external excitant, with chlorate of potash or some other saline, or with the copious exhibition of whey instead of toast-water, in the hope that any such animal fluid absorbed might better supply the fluid and the salts which the blood has lost.

Dane Bank, Holmes Chapel, Aug. 9th, 1848.

\section{TREATMENT OF SCROTAL HERNIA.}

\section{Br JOHN PARKIN, EsQ., M.R.C.S.L. \& L.S.A.}

On Monday evening, August 14th, I was requested to see a little boy, four years of age, who was reported to be suffering intensely from some swelling of the scrotum. I immediately went, and on my arrival found the little fellow laid upon the sofa, crying. His mother informed me that he had been playing about along with some other boys, and had come in a quarter of an hour previous, complaining of pain in the situation of the tumour. She said she thought there had been swelling there before, but the boy had not complained.

On examination, $I$ found the scrotum on the right side enlarged and somewhat tense, from the protrusion of a portion of intestine which had evidently come through the external abdominai ring; the intestine appeared to be filled with fatus. I laid him in the usual position, and then attempted its reduction by the taxis, but was foiled after a trial of a quarter of an hour; I then proceeded to apply cold for some time, and again endeavoured to reduce it, but failed a second time. He was now put into a warm bath, $\left(90^{\circ}\right.$, ) and allowed to remain in it ten minutes or more. During the time he was in, I again essayed its reduction, but with no better success than before; I then ordered him to be taken out, wrapped in a blanket, and laid upon the sofa as before; in a short time afterwards, after again making trial of the taxis, the reduction was speedy and complete. I left, enjoining them to keep him perfectly quiet and still, and to allow him to remain there for the night.

Next morning I found the boy quite well, and ascertained that he had passed a good night.

London, August, 1848 